Fixed Point Theory, 23(2022), No. 1, 179-198

DOI: $10.24193 /$ fpt-ro.2022.1.11

http://www.math.ubbcluj.ro/ nodeacj/sfptcj.html

\title{
A STOCHASTIC PRODUCTION PLANNING PROBLEM
}

\author{
E.C. CANEPA*, D.-P. COVEI** AND T.A. PIRVU*** \\ *University Politehnica of Bucharest, \\ Department of Mathematical Methods and Models, \\ 313 Splaiul Independenței, Bucharest 060042, Romania \\ E-mail: cristinacanepa@yahoo.com \\ ${ }^{* *}$ The Bucharest University of Economic Studies, \\ Department of Applied Mathematics, \\ Piaţa Romană, 1st district, Postal Code: 010374, Postal Office: 22, Romania \\ E-mail: dragos.covei@csie.ase.ro \\ *** McMaster University, Department of Mathematics and Statistics \\ 1280 Main Street West, Hamilton, ON, L8S 4K1, Canada \\ E-mail: tpirvu@math.mcmaster.ca
}

\begin{abstract}
Stochastic production planning problems were studied in several works; the model with one production good was discussed in [3]. The extension to several economic goods is not a trivial issue as one can see from the recent works [8], [9] and [13]. The following qualitative aspects of the problem are analyzed in [9]: the existence of a solution and its characterization through dynamic programming/Hamilton Jacobi Bellman (HJB) equation, as well as the verification (i.e., the solution of the HJB equation yields the optimal production of the goods). In this paper, we stylize the model of [8] and [9] in order to provide some quantitative answers to the problem. This is possible especially because we manage to solve the HJB equation in closed form. We point to a fixed point characterization of the optimal production rates. Among other results, we find that the optimal production rates adjusted for demand are the same across all the goods and they also turn to be independent of some model parameters. Moreover we show that production rates (adjusted for demand) are increasing in the aggregate number of goods produced, and they are also uniformly bounded. Numerical experiments show some patterns of the output.
\end{abstract}

Key Words and Phrases: Stochastic production problem, stochastic control, fixed point.

2020 Mathematics Subject Classification: 35J57, 35J47, 47H10, $60 \mathrm{~J} 70$.

\section{ACKNOWLEDGEMENTS.}

The authors are grateful to the referees and editors for useful remarks that greatly improved the presentation of the paper. This work was supported by a mobility grant of the Romanian Ministery of Research and Innovation, CNCS-UEFISCDI, project number PN-III-P1-1.1-MCD-2019-0151, within PNCDI III, and NSERC grant 5-36700. The second author dedicates this work to the memory of his father, Mathematics Teacher Constantin Covei, who graduated from Babeş-Bolyai University. 


\section{REFERENCES}

[1] O. Alvarez, A quasilinear elliptic equation in $R^{N}$, Proc. Roy. Soc. Edinburgh Sect. A, 126(1996), 911-921.

[2] L. Arnold, Stochastic Differential Equations, Wiley, New York, 1974.

[3] A. Bensoussan, S.P. Sethi, R. Vickson, N. Derzko, Stochastic production planning with production constraints, SIAM J. Control Optim., 22(1984), 920-935.

[4] A. Cadenillas, P. Lakner, M. Pinedo, Optimal control of a mean-reverting inventory, Operations Research, 58(2010), no. 6, 1046-1062.

[5] A. Cadenillas, P. Lakner, M. Pinedo, Optimal production management when demand depends on the business cycle, Operations Research, 61(2013), no. 4, 1046-1062.

[6] D. -P. Covei, T.A. Pirvu, A stochastic control problem with regime switching, Carpathian J. Math., 37 (2021), no 3, 427 - 440

[7] D.-P. Covei, An elliptic partial differential equation modeling the production planning problem, J. Appl. Anal. Comput., 11(2021), no. 2, 903-910.

[8] D.-P. Covei, Symmetric solutions for an elliptic partial differential equation that arises in stochastic production planning with production constraints, Appl. Math. Comput., 350(2019), 190-197.

[9] D.-P. Covei, T.A. Pirvu, An elliptic partial differential equation and its application, Appl. Math. Lett., 101(2020), 1-7.

[10] I. Ekeland, O. Mbodji, T.A. Pirvu, Time consistent portfolio management, SIAM Journal of Mathematical Finance, 3(2012), no.1, 1-32.

[11] I. Ekeland, T.A. Pirvu, Investment and consumption without commitment, Mathematics and Financial Economics, 71(2014), 142-150.

[12] W.H. Fleming, S.P. Sethi, H.M. Soner, An optimal stochastic production planning problem with randomly fluctuating demand, SIAM J. Control Optim., 25(1987), 1494-1502.

[13] A. Gharbi, J.P. Kenne, Optimal production control problem in stochastic multiple-product multiple-machine manufacturing systems, IIE Transactions, 35(2003), 941-952.

[14] J.M. Lasry, P.L. Lions, Nonlinear elliptic equations with singular boundary conditions and stochastic control with state constraints, Math. Ann., 283(1989), 583-630.

[15] T. Leonori, Large solutions for a class of nonlinear elliptic equations with gradient terms, Adv. Nonlinear Stud., 7(2007), 237-269.

[16] T.A. Pirvu, H. Zhang, Investment-consumption with regime-switching discount rates, Math. Social Sci., 71(2014), 142-150.

[17] A. Porretta, Some uniqueness results for elliptic equations without condition at infinity, Commun. Contemp. Math., 5(2003), 705-717.

[18] C.A. Santos, J. Zhou, J.A. Santos, Necessary and sufficient conditions for existence of blow-up solutions for elliptic problems in Orlicz-Sobolev spaces, Complex Var. Elliptic Equ., 62(2017), 887-899.

[19] S.P. Sethi, G.L. Thompson, Applied Optimal Control: Applications to Management Science, Nijhoff, Boston, 1981.

[20] D.G. Simpson, Power Series, NASA Goddard Space Flight Center Greenbelt, Maryland 20771.

[21] G.L. Thompson, S.P. Sethi, Turnpike horizons for production planning, Management Sci., 26(1980), 229-241.

[22] G.L. Thompson, S.P. Sethi, J. Teng, Strong planning and forecast horizons for a model with simultaneous price and production decisions, European Journal of Operational Research, 16(1984), 378-388.

[23] H. Yang, On the existence and asymptotic behavior of large solutions for a semilinear elliptic problem in $\mathbb{R}^{N}$, Commun. Pure Appl. Anal., 4(2005), 187-198.

Received: March 29, 2020; Accepted: May 16, 2020. 